\title{
USO DO VIOLETA DE ALIZARINA N (AVN) COMO REAGENTE ESPECTROFOTOMÉTRICO NA DETERMINAÇÃO DE ALUMÍNIO
}

\author{
Alailson Falcão Dantas, Antônio Celso Spínola Costa e Sérgio Luís Costa Ferreira \\ Instituto de Química - Universidade Federal da Bahia - Br. de Geremoabo, 147, Campus de Ondina - 40170-290 - Salvador - BA
}

Recebido em 6/7/98; aceito em 27/10/99

\begin{abstract}
USE OF THE ALIZARINE VIOLET N (AVN) AS A SPECTROPHOTOMETRIC REAGENT FOR ALUMINIUM DETERMINATION. The present work proposes the application of the 4-Hidroxy-3(2-hydroxynaphtylazo)-benzenesulphonic acid (C.I. 15670), Alizarine Violet $N$ (AVN), as a reagent for direct aluminium determination using molecular absorption spectrophotometry in the presence

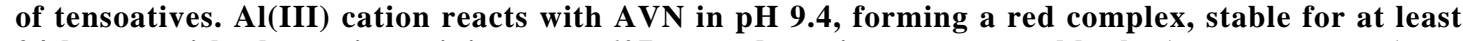
24 hours, with absorption minimum at $607 \mathrm{~nm}$ and, against a reagent blank, $\left(\varepsilon_{\text {complex }}-\varepsilon_{\text {reagent }}\right)=-$ $2.71 \times 10^{4} \mathrm{~L} \cdot \mathrm{mol}^{-1} \cdot \mathrm{cm}^{-1}$. The reaction occurs in the presence of a Triton-X100 and CTAB tensoatives mixture, in the presence of EDTA. AI(III) determination is possible in the linear range of 50 up to 400ng.mL ${ }^{-1}$, with a detection limit of $41 \mathrm{ng} \cdot \mathrm{mL}^{-1}$.
\end{abstract}

Keywords: aluminium; spectrophotometry; tensoatives.

\section{INTRODUÇÃO}

Numerosos métodos espectrofotométricos foram sugeridos para determinação de alumínio e, normalmente, esses métodos não são tão simples, apresentando baixa sensibilidade e seletividade. Os reagentes mais freqüentemente utilizados são a 8-Hidroxiquinolina, Aluminon, Eriocromo Cianine R, Cromoazurol S e Stilbazo ${ }^{1-3}$.

Existem também referências do uso de reagentes tipo $o, o^{\prime}-$ di-hidroxiarilazo como reagentes espectrofotométricos para o alumínio. Florence ${ }^{4}$ apresentou o 5-sulfo-4-dietilamino-2,2'dihidroxiazobenzeno (DDB) para a determinação espectrofotométrica de alumínio na presença de berílio. Woodward e Freiser ${ }^{5}$ propuseram o uso da Calmagita e Ferreira e colaboradores $^{6}$ sugeriram o uso do Azul de Hidroxinaftol (HNB) como reagente para alumínio.

Em espectrofluorimetria, vários reagentes com estrutura semelhante a dos o, $o$ '-di-hidroxiarilazo foram empregados na determinação de alumínio. O Lumogallion foi empregado para determinar alumínio com auxílio de agentes surfactantes ${ }^{7}$ e para a sua determinação fluorimétrica em sangue ${ }^{8}$. O Azul Negro Pontacromo ${ }^{9}$ foi empregado para determinar alumínio em soluções de hemodiálise.

O 1-(2'-hidroxi-5' -sulfobenzeno)-2-naftol, o Violeta de Alizarina N, possui a estrutura molecular ilustrada na Figura 1.

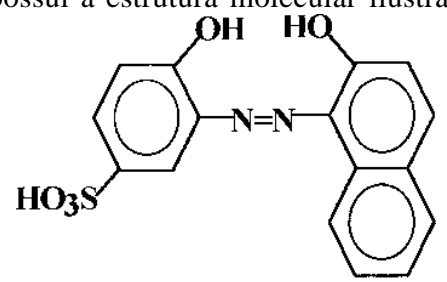

Violeta de Alizarina N (AVN)

Figura 1. Violeta de Alizarina $N(A V N)$.

O AVN foi inicialmente sugerido como um possível reagente fluorescente para alumínio e gálio por Radley ${ }^{10}$, em 1943. Korkisch e Osman ${ }^{11}$ estudaram o uso do AVN para a determinação espectrofotométrica de zircônio. Khalifa e Bishara ${ }^{12}$ verificaram que o AVN formava complexos 2:1 com o vanádio
(IV). Pleniceanu e colaboradores ${ }^{13-14}$ publicaram dois trabalhos envolvendo o uso do AVN como reagente espectrofotométrico. O primeiro, em 1974, sugeria a formação de um complexo $2: 1$ com $\mathrm{V}(\mathrm{V})$ em $\mathrm{pH} 3,1$, o segundo, em 1975, onde o AVN formava complexos 2:1 com W(VI) em pH 2.

A determinação polarográfica do alumínio com o AVN foi bastante estudada ${ }^{15-28}$. Os primeiros a sugerirem esta técnica foram Willard e Dean ${ }^{16}$, onde o complexo AVNAl(III), em $\mathrm{pH} 4,7$, necessitava de 5 horas para se formar em temperatura ambiente.

Também existem algumas referências do uso do AVN em determinações espectrofluorimétricas de alumínio. Após Radley, que foi o primeiro a sugerir o AVN como um possível reagente fluorescente, Weissler e White ${ }^{29}$ indicaram o AVN na determinação de $\mathrm{Al}$ (III) em ligas, observando boa sensibilidade, apesar da sua fluorescência ser destruída por pequenas quantidades de ferro. Bognar e Szabo ${ }^{30}$ fizeram uma comparação entre os reagentes Azul Negro Pontacromo R e o AVN na determinação fluorimétrica de $\mathrm{Al}(\mathrm{III})$.

\section{PARTE EXPERIMENTAL}

\section{Reagentes}

- Solução de Alumínio (III) [1000 $\left.\mu \mathrm{g} . \mathrm{mL}^{-1}\right]$ : preparada pela dissolução de $1,0000 \mathrm{~g}$ de alumínio metálico puro Merck $(99,99 \%)$ em ácido clorídrico $10 \%$ com aquecimento e diluído para $1000 \mathrm{~mL}$ com água desionizada ${ }^{31}$.

- Soluçãa de Alumínio (III) [25 $\mu$ g. $\left.\mathrm{mL}^{-1}\right]$ : preparada pela diluição de uma alíquota de $25,0 \mathrm{~mL}$ da solução de Al(III) $1000 \mu \mathrm{g} . \mathrm{mL}^{-1}$ em balão volumétrico de $1000 \mathrm{~mL}$ com água desionizada.

- Solução do $\boldsymbol{A V N}(\mathbf{0 , 0 4 \% )}$ : preparada pela dissolução de $0,0800 \mathrm{~g}$ do Violeta de Alizarina N (Aldrich), em água desionizada até o volume de $200 \mathrm{~mL}$. Considerando-se os $60 \%$ de pureza indicado pelo fabricante, a concentração analítica desta solução é de $6,6 \times 10^{-4}$ mol.L ${ }^{-1}$

- Solução de CTAB (0,1\%): preparada pela dissolução de $0,1000 \mathrm{~g}$ do brometo de cetiltrimetilamônio Merck em água desionizada até o volume de $100 \mathrm{~mL}$.

- Solução de Triton-X100 (1\%): preparada pela dissolução de $1,00 \mathrm{~g}$ de Triton-X100 Aldrich em água desionizada até o volume de $100 \mathrm{~mL}$. 
- Solução de Triton-X100 (1\%) / CTAB (0,1\%): preparada pela dissolução de $2,50 \mathrm{~g}$ de Triton-X100 Aldrich e de 0,2500g do brometo de cetiltrimetilamônio (CTAB) Merck em água desionizada até o volume de $250 \mathrm{~mL}$.

- Solução tampão amoniacal pH 9,4 (3 mol.L $\left.{ }^{-1}\right)$ : preparada pela dissolução de $70,0 \mathrm{~mL}$ de amônia concentrada $(25 \%)$ e $160,40 \mathrm{~g}$ de cloreto de amônio em água desionizada até o volume de $1000 \mathrm{~mL}$.

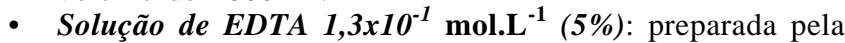
dissolução de $50,00 \mathrm{~g}$ do etileno-diamino-tetra-acetato dissódico Merck em água desionizada até o volume de $1000 \mathrm{~mL}$.

- Solução de KCN 1,5x10 mol.L $^{-1}$ (1\%): preparada pela dissolução de $1,00 \mathrm{~g}$ do cianeto de potássio em água desionizada até o volume de $100 \mathrm{~mL}$.

\section{INSTRUMENTAÇÃO}

- Espectrofotômetro de Absorção Molecular UV-VIS CARY 1E

- Medidor de pH Analyser pH300 com eletrodo de vidro combinado

\section{PROCEDIMENTO GERAL}

Transferiu-se uma alíquota de uma solução padrão de $\mathrm{Al}(\mathrm{III})$ contendo entre 1,25 e $10 \mu \mathrm{g}$ para um balão de $25 \mathrm{~mL}$, e adicionou-se 5,0mL de AVN 0,04\%, 5,0mL de tampão amoniacal $\mathrm{pH}$ 9,4(3 mol. $\left.\mathrm{L}^{-1}\right), 1,0 \mathrm{~mL}$ de EDTA $5 \%$ e $1,0 \mathrm{~mL}$ de Triton-X100 $1 \%$ / CTAB $0,1 \%$ e completou-se o volume com água desionizada. Em seguida, mediu-se a absorvância em $607 \mathrm{~nm}$ contra uma solução de referência contendo os mesmos reagentes adicionados anteriormente, exceto o $\mathrm{Al}(\mathrm{III})$.

\section{RESULTADOS E DISCUSSÃO}

\section{Espectros e estabilidade dos complexos}

$\mathrm{O}$ reagente Violeta de Alizarina $\mathrm{N}$ reage imediatamente com o Al(III), em meio alcalino, na presença de tampão amoniacal $\mathrm{pH} 9,4$, formando um complexo violeta-avermelhado, solúvel em água, que atinge sua absorvância mínima em $607 \mathrm{~nm}$ contra um branco do reagente em presença dos tensoativos TritonX100 e CTAB. A Figura 2 mostra o espectro deste complexo contra sua solução de referência.

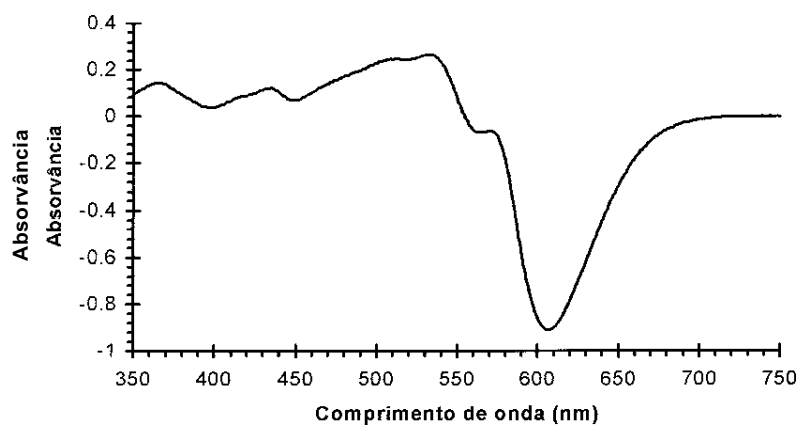

Figura 2. Espectro observado do complexo $A V N-A l(I I I) . A l(I I I)=1 \mu g . m L^{-1}$,

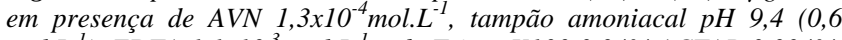
mol.L $\left.L^{-1}\right)$, EDTA 1,1x10 $0^{-3}$ mol. $L^{-1}$ e de Triton-X100 0,04\% / CTAB 0,004\%, contra uma solução de referência (vide procedimento geral).

Observou-se, pelo espectro do sistema AVN-Al(III), que o comprimento de onda de maior diferença entre a absortividade molar do quelato AVN-Al(III) e do AVN e, consequentemente, de maior sensibilidade, apresenta um sinal de absorvância negativo. Este comprimento de onda, onde se mede o consumo do AVN pelo Al(III) para formação do complexo AVN-Al(III), necessariamente não deve ser o comprimento de máxima absorvância do AVN puro, mas sim o de maior diferença de absorvância entre o complexo e o reagente puro.

Observou-se também que o fenômeno se repete para outros complexos do AVN em pH básico, com o $\mathrm{Zn}$ (II), Ni(II) e $\mathrm{Co}$ (II), onde o comprimento de onda de maior diferença entre o quelato e o AVN é o de absorvância mínima, ou seja, absorvância negativa.

Esse fenômeno parece ser comum aos complexos dos reagentes $o, o^{\prime}$-di-hidroxiarilazo, quando estes se formam em pH básico, como pode ser observado no trabalho feito por Amin e colaboradores ${ }^{32}$ onde era medido o sinal de absorvância do consumo do reagente Negro de Eriocromo A, para a formação do seu quelato com o Co. Este mesmo fenômeno foi observado quando os espectro do complexo formado entre o Azul de Hidroxinaftol (HNB) e o alumínio, em meio alcalino, foi obtido experimentalmente no presente trabalho. Este espectro é apresentado na Figura 3.

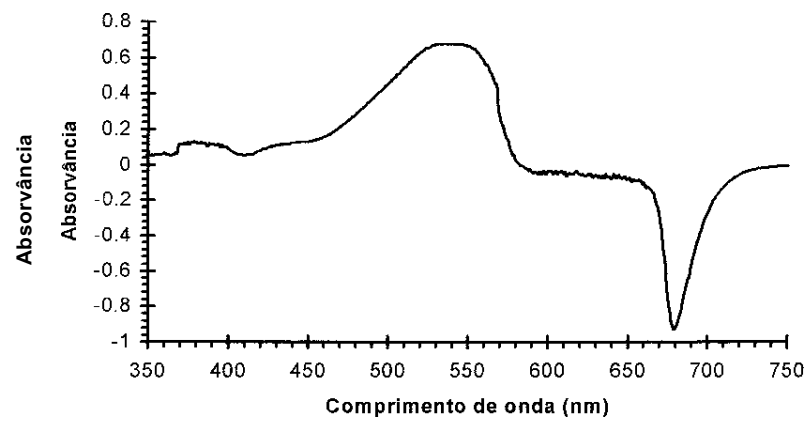

Figura 3. Espectro do Azul de Hidroxinaftol - Al(III) em meio amoniacal. $A l(I I I)=1 \mu \mathrm{g} . \mathrm{mL}^{-1}$, em presença de $H N B 1,3 \times 10^{-4} \mathrm{~mol} . \mathrm{L}^{-1}$, tampão amoniacal pH9,4 0,6 mol.L $L^{-1}$ EDTA $1,1 \times 10^{-3}$ mol. $L^{-1} e$ de Triton-X100 0,04\%/CTAB 0,004\%, contra um branco do HNB.

Os complexos do AVN apresentaram uma alta estabilidade. O sistema AVN-Al(III) é estável por cerca de 24 horas, mesmo na presença de ácidos poliaminocarboxílicos como EDTA e CDTA. A Figura 4 mostra o efeito da variação da absorvância com o tempo para as primeiras 2 horas, na presença de EDTA. Apesar de Pribil e Vesely ${ }^{33}$ afirmarem que o CDTA forma complexos com o alumínio mais rapidamente que o EDTA, a estabilidade do AVN-Al(III) na presença de CDTA $1,1 \times 10^{-3}$ mol.L $\mathrm{L}^{-1}$ também foi testada e observou-se que, para as primeiras 2 horas, a variação da absorvância é inferior a $3 \%$.

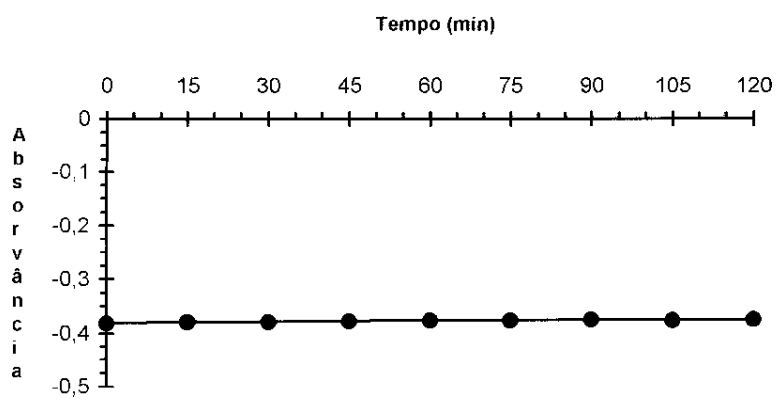

Figura 4. Efeito do tempo sobre a estabilidade do sistema AVN-Al(III). $A l(I I I)=0,4 \mu g . m L^{-1}$, em presença de AVN $1,3 \times 10^{-4}$ mol. $L^{-1}$, tampão

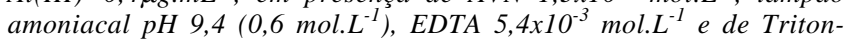
$X 1000,04 \%$ / CTAB 0,004\%, contra uma solução de referência (vide procedimento geral). 


\section{Efeito do pH}

O efeito da variação do $\mathrm{pH}$ sobre a absorvância do complexo AVN-Al(III) foi estudado e os resultados demonstraram que o sistema é muito influenciado pela variação do $\mathrm{pH}$, o que levou ao uso de uma concentração alta $\left(3\right.$ mol.L $\left.\mathrm{L}^{-1}\right)$ do tampão, para evitar grandes flutuações de $\mathrm{pH}$. Os testes foram realizados com tampão amônia/cloreto de amônio 3 mol.L $\mathrm{L}^{-1}$, nas proporções de 1:10 até 10:1. A seguir, na Figura 5, apresenta-se a variação da absorvância com o pH para o sistema AVN-Al(III). Admitindo-se uma flutuação de $5 \%$, a faixa de $\mathrm{pH}$ que leva à maior sensibilidade é de 9,2 a 9,8. Optou-se pelo $\mathrm{pH} 9,4$ para o procedimento geral.

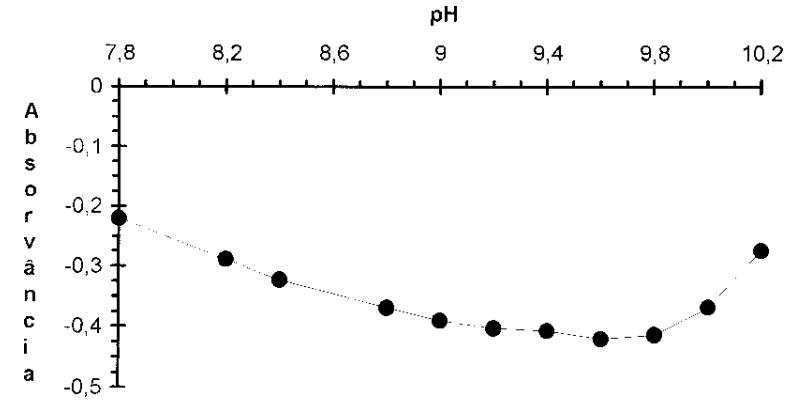

Figura 5. Efeito da variação do $\mathrm{pH}$ sobre a absorvância do sistema $A V N-A l(I I I) . A l(I I I)=0,4 \mu g . m L^{-1}$, em presença de AVN $1,3 \times 10^{-4}$ mol. $L^{-1}$,

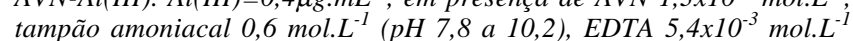
$e$ de Triton-X100 0,04\% / CTAB 0,004\%, contra uma solução de referência (vide procedimento geral).

\section{Efeito da concentração do tampão}

Estudou-se o efeito da variação da concentração da solução tampão amônia/cloreto de amônio, concentração $3 \mathrm{~mol} . \mathrm{L}^{-1}$, e pH 9,4. A variação da concentração de tampão amoniacal não influencia significativamente no sinal de absorvância. Os resultados encontrados são apresentados na Figura 6.

Concentração de tampão amoniacal (mol/L)

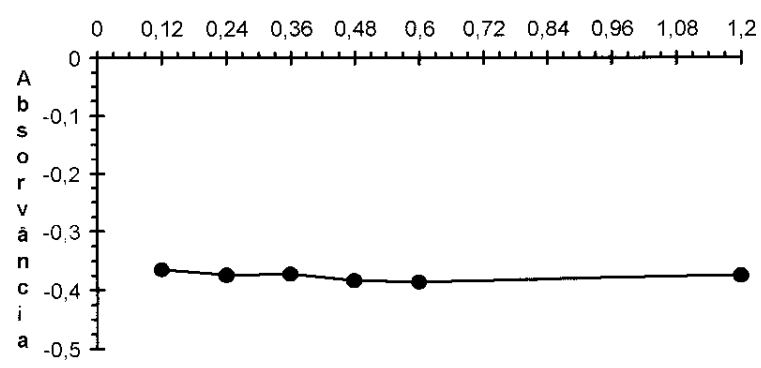

Figura 6. Efeito da variação da concentração de tampão amoniacal sobre a absorvância do sistema $A V N-A l(I I I) . A l(I I I)=0,4 \mu g . \mathrm{mL}^{-1}$, em

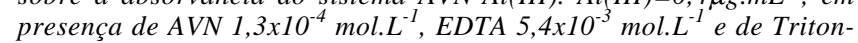
X100 0,04\% / CTAB 0,004\%, contra uma solução de referência (vide procedimento geral).

\section{Efeito da concentração do Violeta de Alizarina N}

O efeito da variação da concentração do Violeta de Alizarina $\mathrm{N}$ foi estudado e observou-se que para uma solução de AVN $6,6 \times 10^{-4}$ mol.L $\mathrm{L}^{-1}$, considerando-se os $60 \%$ de pureza indicados pelo fabricante, a concentração mínima necessária para se obter uma absorvância mínima e constante é de $3 \mathrm{~mL}$. Figura 7 apresenta o gráfico do comportamento do sistema.

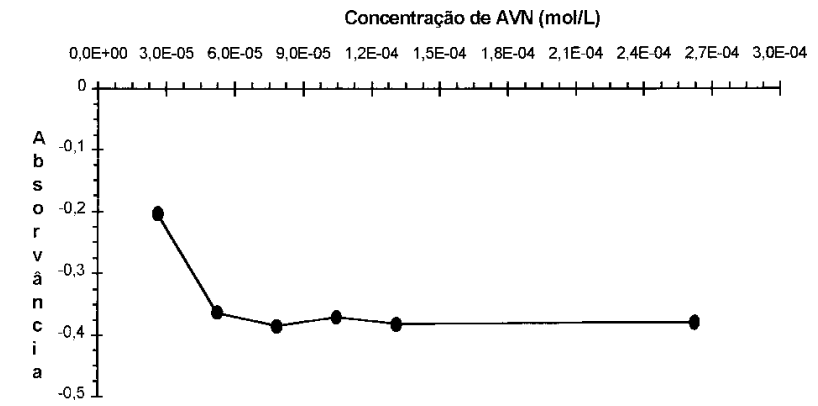

Figura 7. Efeito da variação da concentração de Violeta de Alizarina $N$ sobre a absorvância do sistema $A V N-A l(I I I) . A l(I I I)=0,4 \mu g \cdot \mathrm{mL}^{-1}$, em

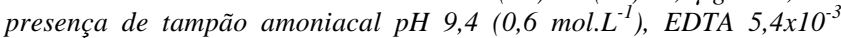
mol. $L^{-1}$ e de Triton-X100 0,04\% / CTAB 0,004\%, contra uma solução de referência (vide procedimento geral).

Adotou-se, como margem de segurança, 5,0 mL da solução do Violeta de Alizarina N $6,6 \times 10^{-4} \mathrm{~mol}^{-L^{-1}}$ no procedimento geral, para garantir um excesso do complexante no meio reacional.

\section{Efeito da concentração dos tensoativos}

Realizaram-se estudos do efeito da variação da concentração de cada tensoativo na mistura e da concentração da mistura sobre a absorvância do sistema AVN-Al(III). Os resultados, para $0,4 \mathrm{mg} . \mathrm{mL}^{-1}$ de $\mathrm{Al}(\mathrm{III})$, são apresentados nas Tabelas $1 \mathrm{e}$ 2 e na Figura 8.

Tabela 1. Efeito da variação da concentração de Triton-X100, na presença de CTAB $0,004 \%(\mathrm{~m} / \mathrm{v})$, sobre a absorvância do sistema AVN-Al(III), para $0,4 \mu \mathrm{g} \cdot \mathrm{mL}^{-1}$ de $\mathrm{Al}(\mathrm{III})$.

\begin{tabular}{cc}
\hline Concentração de Triton-X100 $(\%)$ & Absorvância $(\lambda=607 \mathrm{~nm})$ \\
\hline 0,04 & $-0,389$ \\
0,08 & $-0,402$ \\
0,12 & $-0,408$ \\
0,20 & $-0,399$ \\
0,40 & $-0,420$ \\
\hline
\end{tabular}

Tabela 2. Efeito da variação da concentração de CTAB, na presença de Triton-X100 0,04\% (m/v), sobre a absorvância do sistema AVN-Al(III), para $0,4 \mu \mathrm{g} \cdot \mathrm{mL}^{-1}$ de $\mathrm{Al}(\mathrm{III})$.

\begin{tabular}{cc}
\hline Concentração de CTAB $(\%)$ & Absorvância $(\lambda=607 \mathrm{~nm})$ \\
\hline 0,004 & $-0,387$ \\
0,008 & $-0,406$ \\
0,012 & $-0,429$ \\
0,020 & $-0,447$ \\
0,040 & $-0,471$ \\
\hline
\end{tabular}

Observa-se, pelas Tabelas 1 e 2, que a variação da concentração dos tensoativos influencia a absorvância do sistema AVN-Al(III). Observou-se que é necessário também um excesso de Triton-X100 1\% para evitar o aparecimento de precipitados em solução. De forma preventiva, o Triton-X100 deve ser adicionado antes ou juntamente com o CTAB, sob a forma de uma mistura.

Estudou-se também o efeito da variação da concentração da mistura de tensoativos, tomando-se a proporção de TritonX100/CTAB de 10:1. Este resultado pode ser observado na Figura 8. Embora a figura demonstre que, à medida que se aumenta a concentração da mistura de Triton-X100 1\%/CTAB $0,1 \%$, aumenta-se também o sinal da absorvância do sistema, 


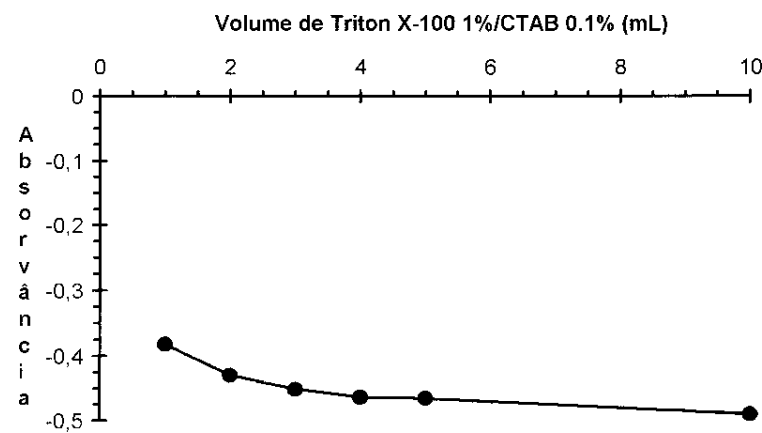

Figura 8. Efeito da variação da concentração da mistura de tensoativos.

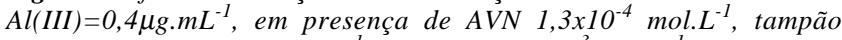
amoniacal pH9,4 $\left(0,6\right.$ mol. $\left.L^{-1}\right)$ e EDTA $5,4 \times 10^{-3}$ mol. $L^{-1}$, contra uma solução de referência (vide procedimento geral).

optou-se pelo volume de 1,0 $\mathrm{mL}$ da mistura dos tensoativos para o procedimento geral porque:

(a) Com esse valor mínimo já se obtém a seletividade desejada

(b) Altas concentrações de tensoativos dificultam o ajuste dos volumes dos balões volumétricos, devido à intensa formação de espuma.

\section{Efeito da concentração de EDTA}

Sendo o Violeta de Alizarina $\mathrm{N}$ um reagente da família dos $o, o$ '-di-hidroxiarilazo, é de se esperar que ele tenha baixa seletividade, principalmente frente aos metais alcalinosterrosos, especialmente os metais cálcio e magnésio. Assim, foi estudado o efeito do EDTA e outros reagentes similares, como o CDTA, EGTA e DTPA, a serem adicionados ao sistema de forma preventiva à ação destes metais.

Pelos resultados apresentados na Tabela 3 e na Figura 9, observa-se que tais agentes mascarantes não alteram significativamente a absorvância do sistema, e, mesmo na presença de altas concentrações de EDTA, não há uma variação significativa do sinal da absorvância do sistema.

Tabela 3. Efeito dos ácidos poliaminocarboxílicos, em concentrações de $1,1 \times 10^{-3}$ mol.L $\mathrm{L}^{-1}$, sobre a absorvância do sistema AVN-Al(III) para $\mathrm{Al}(\mathrm{III})=0,4 \mu \mathrm{g} \cdot \mathrm{mL}^{-1}$.

\begin{tabular}{cc}
\hline Mascarantes & Absorvância $(\lambda=607 \mathrm{~nm})$ \\
\hline Sem mascarante & -0.370 \\
CDTA & -0.354 \\
EDTA & -0.359 \\
EGTA & -0.361 \\
DTPA & -0.363 \\
\hline
\end{tabular}

\section{Características analíticas do método}

A obediência à lei de Beer foi verificada, medindo-se a absorvância do complexo AVN-Al(III) em 607nm, contra um ensaio em branco do AVN, variando-se a concentração do alumínio de 1,25 a $10 \mathrm{mg}$ em $25 \mathrm{~mL}$. Um exemplo de curva analítica encontrada é apresentada na Figura 10, ao qual correspondeu à equação: $\mathrm{A}=-2,71 \times 10^{4} \mathrm{C}_{\mathrm{Al}(\mathrm{III})}(\mathrm{mol} / \mathrm{L})+0,000198$, com um coeficiente de correlação linear $\mathrm{R}=1,00$. Outras características analíticas se encontram nas Tabelas 4 e 5 .

\section{Efeito da ordem de adição dos reagentes}

Estudou-se o efeito da adição dos reagentes envolvidos no procedimento geral. Como o procedimento envolve a adição de
Concentraçäo de EDTA (mol/L)

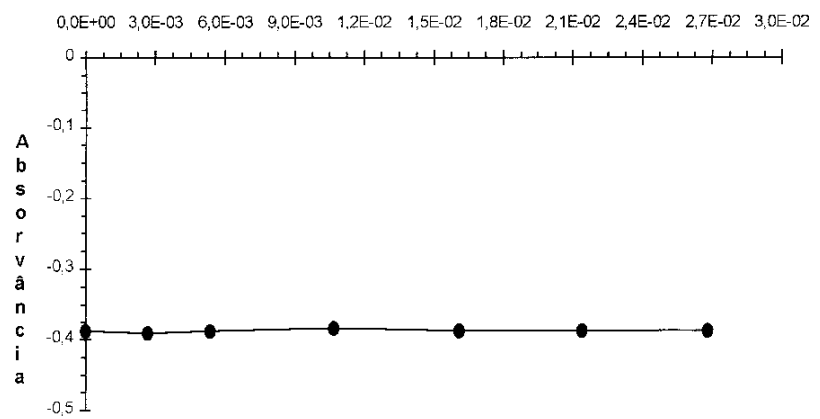

Figura 9. Efeito da variação da concentração de EDTA sobre a absorvância do sistema $A V N-A l(I I I)$. Al(III) $=0,4 \mu g . \mathrm{mL}^{-1}$, em presença

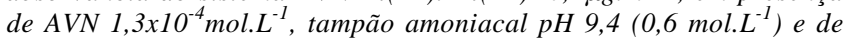
Triton-X100 0,04\% / CTAB 0,004\%, contra uma solução de referência (vide procedimento geral).

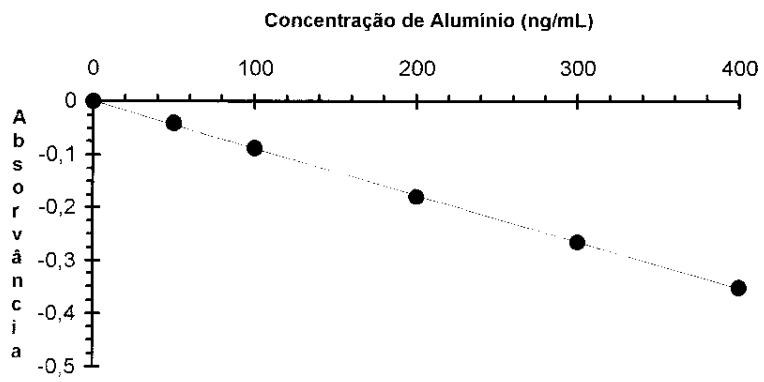

Figura 10. Curva analítica do sistema $A V N-A l(I I I)$. Al(III) $=0$ a $0,4 \mu g . m L^{-1}$, em presença de AVN $1,3 \times 10^{-4}$ mol.L ${ }^{-1}$, tampão

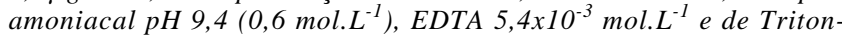
$X 1000,04 \%$ / CTAB $0,004 \%$, contra uma solução de referência (vide procedimento geral).

Tabela 4. Características analíticas do método proposto.

\begin{tabular}{lr}
\hline Absortividade Molar & $-2,71 \times 10^{4} \mathrm{~L} \cdot \mathrm{mol}^{-1} \cdot \mathrm{cm}^{-1}$ \\
Coeficiente de Correlação & 1,00 \\
Limite de Detecção $(3 \sigma)$ & $41 \mathrm{ng} \cdot \mathrm{mL}^{-1}$ \\
Limite de Quantificação $(10 \sigma)$ & $134 \mathrm{ng} \cdot \mathrm{mL}^{-1}$ \\
Faixa Linear Dinâmica & $134 \mathrm{ng} \cdot \mathrm{mL}^{-1}$ a $500 \mathrm{ng} \cdot \mathrm{mL}^{-1}$ \\
$\begin{array}{l}\text { Variação instrumental } \\
\text { do branco }(\mathrm{n}=30)\end{array}$ & $0,31 \%$ \\
\hline
\end{tabular}

Tabela 5. Reprodutibilidade do AVN-Al(III) para $0,4 \mu \mathrm{g} \cdot \mathrm{mL}^{-1}$ de Al(III)

\begin{tabular}{cc}
\hline Média da absorvância & $-0,371$ \\
Desvio padrão & 0,023 \\
Absorvância mínima & $-0,403$ \\
Absorvância máxima & $-0,330$ \\
Número de experimentos (n) & 25 \\
Coeficiente de variação & $6,2 \%$ \\
Intervalo de confiança (95\%) & $-0,371 \pm 0.009$ \\
\hline
\end{tabular}

5 soluções, e haveria muitas combinações a serem testadas, selecionou-se aquelas consideradas mais importantes. Vale salientar que, entre uma adição e outra, executa-se uma rápida agitação, ocorrendo assim um pequeno intervalo de tempo entre as adições. Para os ensaios da Tabela 6 foi utilizado 0,4mg. $\mathrm{mL}^{-1}$ de alumínio.

Pelos resultados obtidos, e registrados na Tabela 6 , observou-se que a ordem de adição altera o sinal da absorvância do 
Tabela 6. Efeito da ordem de adição dos reagentes.

\begin{tabular}{cc}
\hline Ordem de Adição & Absorvância $(\lambda=607 \mathrm{~nm})$ \\
\hline $\mathbf{A l}+\mathbf{R}+\mathbf{T}+\mathbf{D}+\mathbf{M}$ & $-0,399$ \\
$\mathbf{R}+\mathbf{T}+\mathbf{A l}+\mathbf{M}+\mathbf{D}$ & $-0,332$ \\
$\mathbf{A l}+\mathbf{T}+\mathbf{R}+\mathbf{D}+\mathbf{M}$ & $-0,331$ \\
$\mathbf{A l}+\mathbf{D}+\mathbf{T}+\mathbf{R}+\mathbf{M}$ & $-0,209$ \\
$\mathbf{A l}+\mathbf{D}+\mathbf{R}+\mathbf{T}+\mathbf{M}$ & $-0,183$ \\
$\mathbf{R}+\mathbf{T}+\mathbf{D}+\mathbf{M}+\mathbf{A l}$ & $-0,042$ \\
$\mathbf{A l}+\mathbf{M}+\mathbf{R}+\mathbf{T}+\mathbf{D}$ & $-0,037$ \\
$\mathbf{A l}+\mathbf{M}+\mathbf{T}+\mathbf{R}+\mathbf{D}$ & $-0,034$ \\
$\mathbf{A l}+\mathbf{T}+\mathbf{M}+\mathbf{R}+\mathbf{D}$ & $-0,034$ \\
$\mathbf{R}+\mathbf{M}+\mathbf{A l}+\mathbf{T}+\mathbf{D}$ & $-0,033$ \\
$\mathbf{A l}+\mathbf{R}+\mathbf{M}+\mathbf{T}+\mathbf{D}$ & $-0,032$ \\
$\mathbf{M}+\mathbf{T}+\mathbf{A l}+\mathbf{R}+\mathbf{D}$ & $-0,028$ \\
$\mathbf{A l}+\mathbf{D}+\mathbf{M}+\mathbf{R}+\mathbf{T}$ & $-0,024$
\end{tabular}

Al-Al(III), R-AVN, T-Tampão, M-EDTA, D-Tensoativos

sistema AVN-Al(III). Observa-se também que há uma grande diminuição do sinal quando o EDTA é adicionado antes do AVN, quando o $\mathrm{Al}(\mathrm{III})$ é adicionado num meio onde já estejam presentes o EDTA e o AVN ou quando o tampão é adicionado antes do AVN.

\section{Efeito de íons interferentes}

Estudou-se o efeito de vários íons, em diversas proporções, para verificar o grau de interferências dos mesmos sobre o sistema AVN-Al(III). Tomaram-se $10 \mu \mathrm{g}$ de $\mathrm{Al}(\mathrm{III})$, na presença de

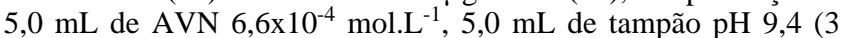
mol. $\left.\mathrm{L}^{-1}\right), 1000 \mathrm{mg}$ do ânion, $1,0 \mathrm{~mL}$ de EDTA $1,3 \times 10^{-1} \mathrm{~mol} . \mathrm{L}^{-1} \mathrm{e}$ $1,0 \mathrm{~mL}$ de Triton-X100 1\% / CTAB 0,1\%, levados a 25mL. Entre os ânions testados estão o citrato, sulfato, tartarato, tiossulfato, fosfato, pirofosfato, cloreto e cianeto numa proporção de 100:1. Nenhum deles apresentou uma alteração significativa na absorvância do sistema AVN-Al(III) e posteriormente foram testados como agentes mascarantes de alguns cátions interferentes.

O Violeta de Alizarina $\mathrm{N}$ não é um reagente específico para o alumínio, o que obrigou o uso de agentes mascarantes para aumentar a sua seletividade. Para o estudo da interferência dos cátions tomaram-se $10 \mu \mathrm{g}$ de $\mathrm{Al}(\mathrm{III}), 100-2000 \mu \mathrm{g}$ do cátion in-

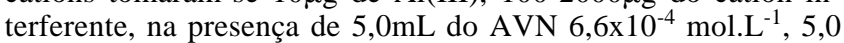
$\mathrm{mL}$ de tampão $\mathrm{pH} 9,4$ (3 mol. $\left.\mathrm{L}^{-1}\right), 1,0 \mathrm{~mL}$ de EDTA $1,3 \times 10^{-1}$ mol. $\mathrm{L}^{-1}$ e $1,0 \mathrm{~mL}$ de Triton-X100 1\%/CTAB 0,1\%, levados a 25 mL. Observou-se que $\mathrm{Pb}(\mathrm{II})$ (200:1), W(VI), $\mathrm{Mo}(\mathrm{VI}), \mathrm{Hg}(\mathrm{II})$, $\mathrm{Na}(\mathrm{I})$ e $\mathrm{K}(\mathrm{I})$ (100:1), $\mathrm{Ca}(\mathrm{II}), \mathrm{Mg}(\mathrm{II})$ e $\mathrm{Ba}(\mathrm{II})$ (50:1), Bi(III), $\mathrm{Sb}$ (III) e $\mathrm{Sr}$ (II) (10:1) não apresentam interferência sobre o sinal de absorvância do sistema AVN-Al(III), na presença de EDTA $5,4 \times 10^{-3}$ mol.L ${ }^{-1}$. Observou-se também que o $\mathrm{Zn}(\mathrm{II})$ e $\mathrm{Cd}(\mathrm{II})$ (20:1) não apresentam interferência sobre o sinal de absorvância do sistema AVN-Al(III) em presença de EDTA $5,4 \times 10^{-3}$ mol. $\mathrm{L}^{-}$

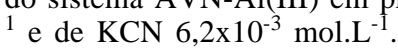

Porém, para os mesmos 10mg de $\mathrm{Al}(\mathrm{III})$, os cátions $\mathrm{Ni}(\mathrm{II})$, Cr(III), Co(II), Ti(IV), Cu(I), Cu(II), Fe(II), Fe(III), Sn(IV), $\mathrm{Be}(\mathrm{II}), \mathrm{La}(\mathrm{III}), \mathrm{Mn}(\mathrm{II}), \mathrm{V}(\mathrm{IV})$ e V(V) (10:1) apresentaram interferência sobre o sinal de absorvância do sistema AVN$\mathrm{Al}(\mathrm{III})$, mesmo na presença de EDTA $5,4 \times 10^{-3}$ mol. $\mathrm{L}^{-1}$ e dos agentes mascarantes $\mathrm{KCN}$, citrato, tartarato, fosfato, CDTA, ácido ascórbico e ácido tioglicólico em concentrações em torno de 0,01 mol. $\mathrm{L}^{-1}$.

\section{Aplicação}

O procedimento proposto foi testado na determinação de alumínio em amostras padrões de ligas e uma amostra de calcário. Para cada análise foi construída uma curva analítica, conforme o procedimento descrito no procedimento geral, e 5 replicatas $(n=5)$ para cada amostra. Foram adicionados agentes mascarantes, variando-se as concentrações e a natureza dos mesmos, de acordo com os interferentes encontrados nas amostras. A abertura das amostras foi realizada tomando-se entre $0,01 \mathrm{~g}$ a $0,1 \mathrm{~g}$ da amostra, $10 \mathrm{~mL}$ de $\mathrm{HCl}$ concentrado, aquecimento, e depois levado a $100 \mathrm{~mL}$. Os resultados encontrados são descritos na Tabela 7 .

Tabela 7. Determinação de alumínio em amostras padrões.

\begin{tabular}{cc}
\hline Amostras & $\% \mathrm{Al}(\mathrm{III})$ encontrada $(\mathrm{n}=5)$ \\
\hline Liga de $\mathrm{Zn} \mathrm{94b}$ & $4,26 \pm 0.12$ \\
Liga de Mg 171 & $3,17 \pm 0.09$ \\
Calcário & $2,06 \pm 0.29$ \\
\hline
\end{tabular}

As ligas analisadas são padrões do NIST, e contém a seguinte composição: Liga de Zn $94 \boldsymbol{b}$ - $\mathrm{Al}$ (4,07\%), $\mathrm{Cu}$ (1,01\%), $\mathrm{Mg}(0,042 \%), \mathrm{Fe}(0,018 \%), \mathrm{Mn}(0,014 \%), \mathrm{Pb}(0,006 \%), \mathrm{Ni}$ $(0,006 \%), \mathrm{Sn}(0,006 \%)$ e Cd $(0,002 \%)$. Liga de Mg $171-\mathrm{Al}$ $(2,98 \%), \mathrm{Zn}(1,05 \%), \mathrm{Mn}(0,45 \%)$, Si $(0,012 \%), \mathrm{Cu}(0,011 \%)$, $\mathrm{Pb}(0,0033 \%), \mathrm{Fe}(0,0018 \%)$ e $\mathrm{Ni}(0,0009 \%)$.

A amostra de calcário, contendo Al (2,03\%), foi analisada também por absorção atômica e comparou-se as médias obtidas.

\section{CONCLUSÕES}

O principal objetivo deste trabalho foi o estudo do Violeta de Alizarina N (AVN) como um reagente espectrofotométrico para a determinação do alumínio, e o desenvolvimento de um procedimento analítico para a determinação de traços do mesmo. Com base nos resultados obtidos conclui-se que:

- Apesar da sua pouca seletividade, o Violeta de Alizarina N (AVN) apresentou-se como um reagente espectrofotométrico muito sensível, e entre as suas virtudes destacam-se a alta solubilidade do reagente e seus complexos em solução aquosa, e sua estabilidade como uma solução aquosa quando estocado em recipiente escuro, ao contrário de regentes similares.

- A baixa pureza do AVN comercial, muito comum aos reagentes da família dos $o, o^{\prime}$-di-hidroxiarilazo compostos, não representou nenhuma dificuldade no desenvolvimento de procedimentos espectrofotométricos com este reagente.

- Os tensoativos apresentam uma grande influência sobre os espectros do AVN e seus complexos, melhorando a resolução espectral dos mesmos e aumentando a sensibilidade e seletividade de alguns sistemas.

- É possível a determinação de alumínio operando-se numa faixa de $\mathrm{pH}$ de 9,2 até 9,8, na presença da mistura de tensoativos Triton-X100/CTAB.

- O sistema AVN-Al(III), em meio básico, apresenta-se como um sistema com alta sensibilidade, comparável a métodos envolvendo outros $o, o^{\prime}$-di-hidroxiarilazo, como a Calmagita e o Azul de Hidroxinaftol, ou métodos envolvendo técnicas fluorimétricas para o mesmo sistema AVN-Al(III). O sistema apresenta uma sensibilidade superior à obtida com o mesmo reagente em métodos envolvendo técnicas polarográficas, onde o sistema foi muito estudado.

- O sistema AVN-Al(III), em pH básico, forma-se com uma velocidade de reação muito maior que as apresentadas em sistemas fluorimétricos ou polarográficos. A reação é instantânea e a absorvância é estável por cerca de 24 horas, ao contrário de outras técnicas onde as medidas são feitas após períodos de 1 a 3 horas.

- A ordem de adição dos reagentes é muito importante e o EDTA, ou outro mascarante, somente deve ser adicionado após a formação do complexo AVN-Al(III).

- A variação da concentração de cada tensoativo, ou de sua mistura, exercem influência sobre o sinal de absorvância do complexo. 


\section{AGRADECIMENTOS}

Este trabalho é parte integrante de uma monografia de mestrado, desenvolvida e aprovada pelo programa de pós-graduação do Instituto de Química da Universidade Federal da Bahia, com bolsa de estudos financiada pelo CNPq.

\section{REFERÊNCIAS}

1. Snell, F. D.; Photometric and Fluorimetric Methods Analysis; Wiley, Ed.; Nova Iorque, 1978; 1, p 585.

2. Marczenko, Z.; Spectrophotometric Determination of Elements; Horwood, Ed.; Inglaterra, 1976; p 110.

3. Ueno, K., Imamura, T.; Cheng, K. L.; Handbook of Organic Analytical Reagents; CRC Press, Ed.; Florida, 1992, 2a . Edição.

4. Florence, T. M.; Anal. Chem. 1965, 37, 704.

5. Woodward, C.; Freiser, H.; Talanta 1968, 15, 321.

6. Ferreira, S. L. C.; Dantas, A. F.; Leite, N. O.; Costa, A. C. S.; de Andrade, J. B.; Talanta 1994, 41, 1631.

7. Ishibashi, N.; Kina, K.; Anal. Lett. 1972, 5, 637.

8. Suzuki, Y.; Imai, S.; Kamiki, T.; Analyst 1989, 114, 839.

9. Raggi, M. A.; Varani, G.; Cavrini, V.; Lacché, D.; Nobile, L.; Anal. Lett.; 1986, 19, 1435.

10. Radley, J. A.; Analyst 1943, 68, 369; Chem. Abs. 1944, 38, No.930-9.

11. Korkish, J.; Osman, M.; Z. Anal. Chem. 1959, 171, 107; Anal. Abs. 1960, 07, No. 2673.

12. Khalifa, H.; Bishara, S. W.; Z. Anal. Chem. 1961, 179, 345.

13. Pleniceanu, M.; Burnea, L.; Revue roum. Chim. 1974, 19, 501; Anal. Abs. 1974, 27, No. 2543.

14. Pleniceanu, M.; Burnea, L.; Revue roum. Chim. 1975, 20 , 267; Anal. Abs. 1975, 28, No. 4B179.
15. Dean, J. A.; Bryan, H. A.; Anal. Chim. Acta. 1957, 16, 87. 16. Willard, H. H.; Dean, J. A.; Anal. Chem. 1960, 22, 1264.

17. Dean, J. A.; Bryan, H. A.; Anal. Chim. Acta. 1957, 16, 94.

18. Perkins, M.; Reynolds, G. F.; Anal. Chim. Acta. 1958, $18,616$.

19. Perkins, M.; Reynolds, G. F.; Anal. Chim. Acta. 1958, $18,625$.

20. Perkins, M.; Reynolds, G. F.; Anal. Chim. Acta. 1958 19, 54.

21. Perkins, M.; Reynolds, G. F.; Anal. Chim. Acta. 1958, 19, 194.

22. Latimer, G.W.; Talanta 1968, 15, 01.

23. Stryjewska, E.; Rubel, S.; Electroanalysis 1991, 3, 995

24. Farias, P. A. M.; Takase, I.; Electroanalysis 1992, 4, 823

25. Stryjewska, E.; Rubel, S.; Kusmierczyk, K.; Chemia Analityczna 1992, 37, 43

26. Downard, A. J.; Powell, H. K. J.; Xu, S. H.; Anal. Chim. Acta. 1992, 262, 339

27. Ma, M. H.; Johnson, K. E.; J. Electroanal. Chem. 1993 335, 97

28. Paneli, M.G.; Vulgaropoulos, A.; Electroanalysis 1993 5,355

29. Weissler, A.; White, C. E.; Ind. Eng. Chem. 1946, 18, 530.

30. Bognar, J.; Szabo, M. P.; Mikrochim. Acta 1969, 221; Anal. Abs. 1970, 18, No. 2281

31. Bruno, T. J.; Svoronos, P. D. N.; CRC Handbook of Basic Tables for Chemical Analysis; CRC Press, Ed.; Flórida; 1996; p 364.

32. Amin, A. A. M.; El Raheem, A. A. A; Ozman, F. A.; Z Anal. Chem. 1959, 167, 8; Anal. Abs. 1960, 7, No 149.

33. Pribil, R.; Veselý, V.; Talanta 1962, 9, 23. 